\title{
Towards an Experimental Framework for Measuring Usability of Model-Driven Tools ${ }^{*}$
}

\author{
Jose Ignacio Panach ${ }^{1}$, Nelly Condori-Fernández ${ }^{2}$, \\ Arthur Baars ${ }^{1}$, Tanja $\operatorname{Vos}^{1}$, Ignacio Romeu ${ }^{1}$, and Óscar Pastor ${ }^{1}$ \\ ${ }^{1}$ Centro de Investigación en Métodos de Producción de Software ProS, \\ Universitat Politècnica de València, \\ Camino de Vera s/n, 46022 Valencia, Spain \\ \{jpanach, abaars, tvos, iromeu, opastor\} @pros.upv.es \\ ${ }^{2}$ Faculty of Electrical Engineering, Mathematics and Computer Science, \\ University of Twente, \\ Information Systems Group, \\ 7500 AE Enschede, P.O. Box 217, The Netherlands \\ nelly@pros.upv.es
}

\begin{abstract}
According to the Model-Driven Development (MDD) paradigm, analysts can substantially improve the software development process concentrating their efforts on a conceptual model, which can be transformed into code by means of transformation rules applied by a model compiler. However, MDD tools are not widely used in industry. One of the reasons for this poor adoption is the lack of usability of MDD tools. This paper presents a framework to evaluate the usability of such tools. The framework will be used as a basis for a family of experiments to get clear insights into the barriers to usability that prevent MDD tools from being widely adopted in industry.
\end{abstract}

Keywords: Usability, model-driven development, evaluation framework.

\section{Introduction}

MDD tools can offer many benefits for a software developing company: reduced costs, reduced development time, higher quality and higher customer satisfaction [7]. However, the wide acceptance of MDD is not yet a reality. One reason for this situation is the lack of usability of MDD tools. The main contribution of this paper is an empirical framework to perform a set of usability evaluations for MDD tools. By experimental framework we mean the definition of a process and the elements involved in it with the aim of performing evaluations unambiguously. The framework will be used to perform the evaluation of several MDD tools knowing clearly the required elements and the stages to perform the experiment.

Several authors such as Fiora [2] and Kostiainen [5] have proposed experimental frameworks for measuring system usability. If we focus our research on MDD tools,

This work has been developed with the support of MICINN, GVA and ITEA 2 call under the projects PROS-Req (TIN2010-19130-C02-02), ORCA (PROMETEO/2009/ 015) and UsiXML (20080026). 
little work has been published about their usability. We can only find usability frameworks defined for evaluating usability in Computer Aided Software Engineering (CASE) tools in general, such as the work of Senapathi [8]. However, frameworks defined for CASE tools are not always valid for MDD tools. In contrast to conventional CASE tools that are oriented to software development based on design and programming, MDD tools have to cope with specific features where the modeling and the programming perspective become intertwined. MDD tools have as peculiarity that they have an underlying software delevopment method which must be easily understandable to the user. Studying related work, we conclude that more work must be done on the usability evaluation of MDD tools to become fully accepted in industry. The paper is divided into these sections: 2nd describes the framework, 3th applies the framework to a specific MDD tools, and 4th presents the conclusions.

\section{Empirical Framework to Evaluate the Usability of MDD Tools}

The empirical framework is composed of a usability evaluation model and an experimental process, which have been designed using Wohlin's proposal [9].

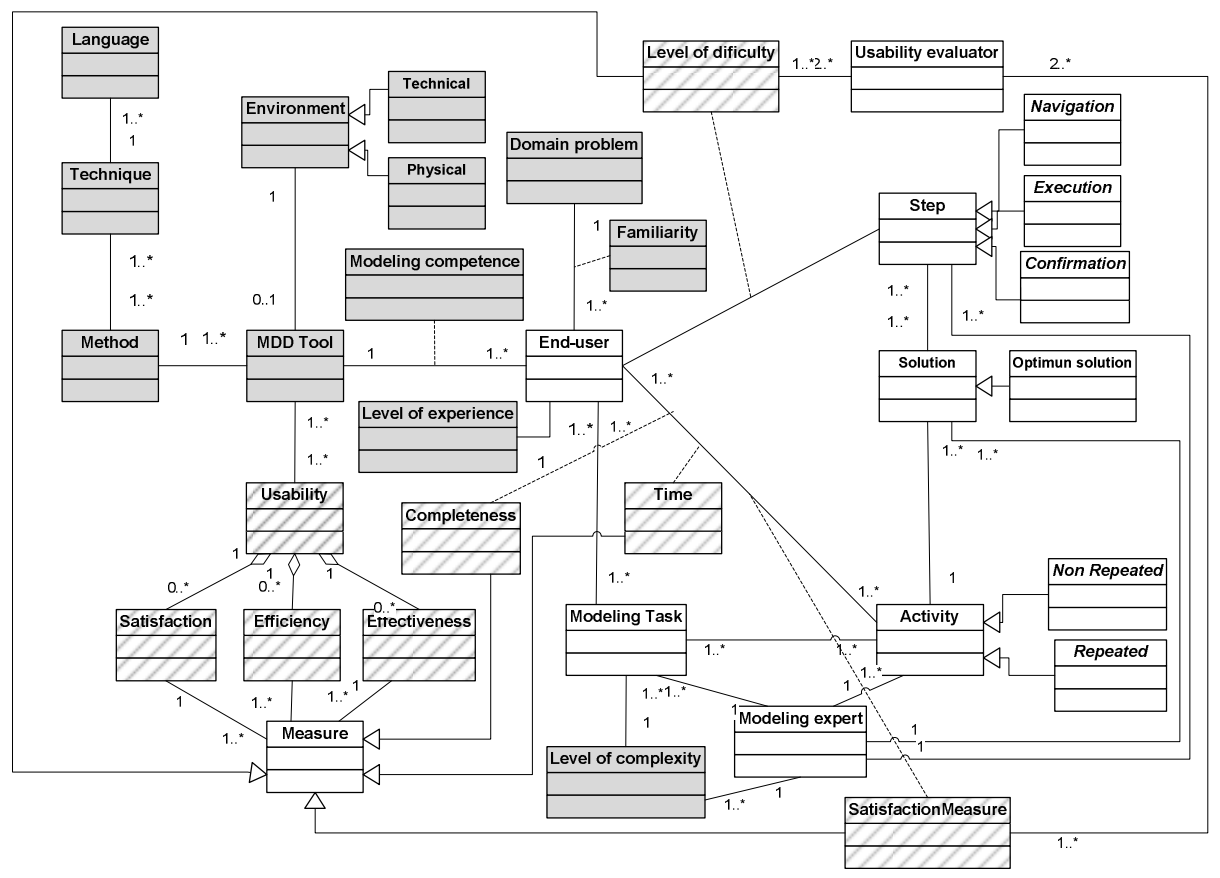

Fig. 1. Usability Evaluation Model

The usability evaluation model identifies the most relevant elements for evaluating the usability of MDD tools (Figure 1). According to Figure 1, we aim to evaluate the usability of MDD tools by means of: satisfaction, efficiency and effectiveness, such as ISO 9241-11 proposes [4]. In summary, this model represents: 
the method that underpins the MDD tool, the end-user features, and the activities that the end-user will execute in the experiment. Classes with a grey background represent elements that can be changed and controlled in the experiment and classes with a background of diagonal lines represent elements that can be quantified with one or more measures.

The experimental process focuses on the stages that compose the process to perform the usability evaluation according to the usability model. There are four stages [9] (Figure 2): (1) Definition: This determines the foundation of the experiment; (2) Planning: This specifies how the experiment is conducted. (3) Operation: In this step, the researcher performs the experiment and collects the needed data; (4) Analysis: In this step, the researcher interprets the experiment data. Each one of these stages is divided into substages, such as Figure 2 shows.

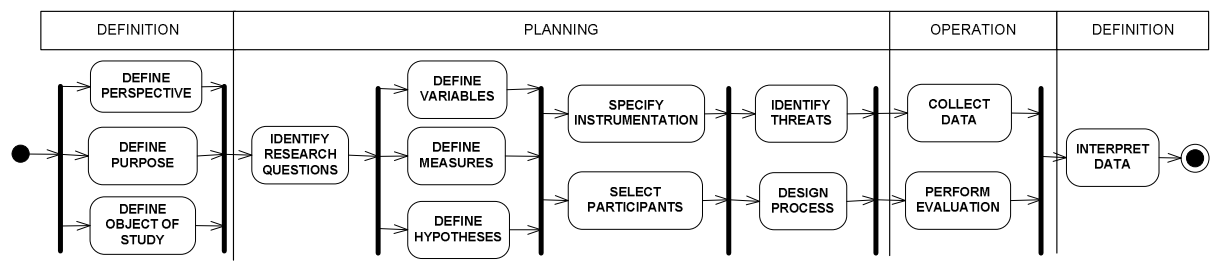

Fig. 2. Process to evaluate usability

\section{Applying the Usability Framework to a Specific MDD Tool}

This section explains the usability evaluation that we performed with the proposed framework applied to an MDD tool called OLIVANOVA [1]. We focus our study on effectiveness and efficiency, relegating the satisfaction to a future experiment. Next, for space reason, we detail only some elements that compose the Planning stage (Figure 2): hypotheses, variables and participants. However, it is important to note that the Usability Evaluation Model (Figure 1) is instantiated throughout all the substages that compose the Definition and Planning stages (Figure 2).

We have one null hypothesis for efficiency and another for effectiveness:

- $\mathbf{H}_{\mathbf{1 0}}$ : When using OLIVANOVA for modeling tasks with different levels of complexity, the efficiency is the same independently of users' experience.

- $\mathbf{H}_{\mathbf{2 0}}$ : When using OLIVANOVA for modeling tasks with different levels of complexity, the effectiveness is the same independently of users' experience.

We have identified dependent variables and independent variables [9]:

- Dependent variables: Efficiency and effectiveness. Efficiency was measured by task completion percentage in relation to the time spent to perform a task. Effectiveness is the level of completeness reached in every task. This variable was calculated by two measures: 1) the percentage of tasks carried out correctly; 2) the percentage of correctly performed activities that were carried out optimally (without any difficulty compared to experts).

- Independent variables: The level of complexity of the tasks, the modeling competence, and the level of experience using MDD tools. 


\section{We used three groups of participants:}

- Type I (Experts): Experienced using the evaluated tool. This group was composed of researchers of the ProS center of the Technical University of Valencia.

- Type II (Medium): Experienced using similar tools. This group was recruited from the regional Valencian public administration who are familiar with open source MDD tools like Moskitt [6].

- Type III (Novice): No experience with the tool nor with similar tools. This group was composed of engineers from the Technological Institute of Computer Science who are familiar with Object-Oriented concepts but not with any modeling tool.

We used four users from each group to work with a balanced number of users. Instruments used to perform the experiment can be found at [3]. Once the experiment was finished, we analyzed the results of the efficiency and the effectiveness. Efficiency was measured by task completion percentage in relation to the time spent doing a task. According to an ANOVA test, we reject the null hypothesis. However, if this analysis is carried out excluding the group of experts, there are no differences in the mean efficiency scores with the other two types of users. Effectiveness was measured in terms of modeling task completion percentage and percentage of correct tasks that were carried out optimally. With the ANOVA test, we found significant differences for both the tasks of medium level of difficulty and high level of difficulty in the three groups of users. Therefore, we conclude that $\mathrm{H}_{20}$ is not satisfied.

\section{Conclusions and Future Work}

This paper proposes an empirical framework to evaluate the usability of MDD tools. The framework aims to replicate the usability evaluation of MDD tools in similar conditions. With the purpose of evaluating our framework; it was applied to a MDD tool called OLIVANOVA. The results of the evaluation demonstrate that changes must be applied to OLIVANOVA to improve the users' effectiveness and efficiency. As future work, we want to repeat our usability test with more users and considering the user's satisfaction. Moreover, we want to assess the framework with other MDD tools different from OLIVANOVA.

\section{References}

1. CARE Technologies S.A, http: / / www . care-t. com

2. Fiora, T.W., Au, B.S., Warren, I., Dobbie, G.: Automated Usability Testing Framework. In: Proc. 9th Australasian User Interface Conference, AUIC 2008 (2008)

3. Instruments, http://hci.dsic.upv.es/testOLIVANOVA/

4. ISO 9241-11 Ergonomic requirements for office work with visual display terminals (1998)

5. Kostiainen, K., Uzun, E.: Framework for Comparative Usability Testing of Distributed Applications. In: Security User Studies: Methodologies and Best Practices Workshop (2007)

6. Moskitt, http: //www. moskitt.org/

7. Selic, B.: The Pragmatics of Model-Driven Development. IEEE Software 20, 19-25 (2003)

8. Senapathi, M.: A Framework for the Evaluation of CASE Tool Learnability in Educational Environments. Journal of Information Technology Education 4, 61-84 (2005)

9. Wohlin, C., Runeson, P., Höst, M.: Experimentation in Software Engineering: An Introduction. Springer, Heidelberg (1999) 